\title{
AN X-RAY-SELECTED SAMPLE OF CANDIDATE BLACK HOLES IN DWARF GALAXIES
}

\author{
Sean M. Lemons ${ }^{1}$, Amy E. Reines ${ }^{1,3}$, Richard M. Plotkin ${ }^{1}$, Elena Gallo ${ }^{1}$, and Jenny E. Greene ${ }^{2}$ \\ ${ }^{1}$ Department of Astronomy, University of Michigan, 1085 South University Ave., Ann Arbor, MI 48109, USA; reines@umich.edu \\ ${ }^{2}$ Department of Astrophysical Sciences, Princeton University, Princeton, NJ 08544, USA \\ Received 2014 December 20; accepted 2015 February 20; published 2015 May 14
}

\begin{abstract}
We present a sample of hard X-ray-selected candidate black holes (BHs) in 19 dwarf galaxies. BH candidates are identified by cross-matching a parent sample of $\sim 44,000$ local dwarf galaxies $\left(M_{\star} \leqslant 3 \times 10^{9} M_{\odot}, z<0.055\right)$ with the Chandra Source Catalog and subsequently analyzing the original X-ray data products for matched sources. Of the 19 dwarf galaxies in our sample, eight have X-ray detections reported here for the first time. We find a total of 43 point-like hard X-ray sources with individual luminosities $L_{2-10 \mathrm{keV}} \sim 10^{37}-10^{40} \mathrm{erg} \mathrm{s}^{-1}$. Hard X-ray luminosities in this range can be attained by stellar-mass X-ray binaries (XRBs) and by massive BHs accreting at low Eddington ratio. We place an upper limit of 53\% (10/19) on the fraction of galaxies in our sample hosting a detectable hard X-ray source consistent with the optical nucleus, although the galaxy center is poorly defined in many of our objects. We also find that $42 \%$ (8/19) of the galaxies in our sample exhibit statistically significant enhanced hard X-ray emission relative to the expected galaxy-wide contribution from low-mass and high-mass XRBs, based on the $L_{2-10 \mathrm{keV}}^{\mathrm{XRB}}-M_{\star}-$ star formation rate relation defined by more massive and luminous systems. For the majority of these X-ray-enhanced dwarf galaxies, the excess emission is consistent with (but not necessarily due to) a nuclear X-ray source. Follow-up observations are necessary to distinguish between stellarmass XRBs and active galactic nuclei powered by more massive BHs. In any case, our results support the notion that X-ray-emitting BHs in low-mass dwarf galaxies may have had an appreciable impact on reionization in the early universe.
\end{abstract}

Key words: galaxies: active - galaxies: dwarf - galaxies: nuclei $-\mathrm{X}$-rays: binaries $-\mathrm{X}$-rays: galaxies

\section{INTRODUCTION}

It is now well established that massive black holes (BHs) reside in the nuclei of essentially every giant galaxy with a bulge (e.g., Kormendy \& Ho 2013), yet the incidence of such $\mathrm{BHs}$ in dwarf galaxies is unknown. Determining the occurrence of massive BHs in present-day low-mass galaxies and studying their properties is currently our best observational probe of the BH seed population in the early universe (Volonteri 2010; Greene 2012; Natarajan 2014). At present, dynamical detections of small BHs in distant dwarf galaxies are not feasible, and so we must search for accreting BHs that shine as active galactic nuclei (AGNs). Studying the X-ray radiation from massive BHs in dwarf galaxies is particularly important for models of reionization of hydrogen at very high redshifts (e.g., Volonteri \& Gnedin 2009).

Over the past decade, systematic searches using optical spectroscopy from the Sloan Digital Sky Survey (SDSS) have revealed hundreds of low-mass AGNs. The first such studies focused on finding broad-line AGNs with virial BH masses $M_{\mathrm{BH}} \lesssim 2 \times 10^{6} M_{\odot}$ (Greene \& Ho 2004, 2007) and narrowline AGNs in galaxies with absolute $g$-band magnitudes $M_{g} \gtrsim-20 \mathrm{mag}$ (Barth et al. 2008). More recently, Reines et al. (2013) pushed to even lower BH and galaxy masses by searching the spectra of dwarf galaxies with stellar masses $M_{\star} \lesssim 3 \times 10^{9} M_{\odot}$ for both broad- and narrow-line signatures of active BHs.

The optically selected samples are likely just the tip of the iceberg as they are biased toward relatively high Eddington ratios and toward galaxies with little ongoing star formation. High-resolution X-ray and radio observations offer powerful

\footnotetext{
${ }^{3}$ Hubble Fellow.
}

alternatives for discovering massive BHs in low-mass galaxies (e.g., Gallo et al. 2008, 2010; Reines et al. 2011, 2014; Reines \& Deller 2012; Miller et al. 2012; Schramm et al. 2013).

Here we aim to find new massive BH candidates in dwarf galaxies by making use of a wealth of archival data from the Chandra X-ray Observatory. With its low background and ability to resolve point sources from diffuse X-ray emission, Chandra can detect accretion signatures from massive BHs (even nearly quiescent ones) that may not be accessible at other wavebands (Soria et al. 2006; Ghosh et al. 2008; Zhang et al. 2009; Gallo et al. 2010; Pellegrini 2010).

This work bears technical similarities to searches for ultraluminous X-ray sources (ULXs) in dwarf galaxies (e.g., Swartz et al. 2008; Kaaret et al. 2011; Prestwich et al. 2013; Plotkin et al. 2014; Brorby et al. 2014). ULXs are off-nuclear $\mathrm{X}$-ray sources in excess of the Eddington limit for a $10 M_{\odot} \mathrm{BH}$ $\left(\sim 10^{39} \mathrm{erg} \mathrm{s}^{-1}\right)$, the majority of which are now thought to be luminous high-mass-X-ray binaries (XRBs). Luminosities in this regime, however, are also commonly produced by massive BHs accreting at low Eddington ratios (Ho 2009).

This paper is organized as follows. Our sample selection is outlined in Section 2. The Chandra data reduction and photometry are described in Section 3. In Section 4, we present our analysis and results. A summary of our conclusions and a brief discussion are given in Section 5.

\section{SAMPLE SELECTION}

We construct our parent sample of dwarf galaxies using stellar masses provided by the NASA-Sloan Atlas ${ }^{4}$ (NSA) of local $(z \leqslant 0.055)$ galaxies. The NSA provides image mosaics,

\footnotetext{
http://www.nsatlas.org.
} 
photometry and various galaxy parameters based on a reanalysis of optical and ultraviolet observations from the SDSS and the Galaxy Evolution Explorer (GALEX). Galaxy stellar masses in the NSA are derived from the kcorrect code of Blanton \& Roweis (2007). Masses are given in units of $M_{\odot} h^{-2}$, and we adopt $h=0.73$. To select dwarf galaxies, we impose a stellar mass upper limit of $M_{\star} \leqslant 3 \times 10^{9} M_{\odot}$, which is approximately equal to the stellar mass of the Large Magellanic Cloud. Our mass threshold is identical to that used by Reines et al. (2013) in their optical spectroscopic search for active massive BHs, leaving us with 44,594 objects.

After selecting dwarf galaxies from the NSA, we crossmatch our parent sample to the list of X-ray sources within the Chandra Source Catalog (CSC, Release 1.1; Evans et al. 2010). The CSC includes point and compact $\left(\lesssim 30^{\prime \prime}\right)$ sources detected in the Advanced CCD Imaging Spectrometer (ACIS) and High Resolution Camera imaging observations from approximately the first eight years of Chandra operations, and a source must have a flux at least three times larger than its uncertainty to be included in the CSC (see Evans et al. 2010). Using a match radius of $5^{\prime \prime}$ from the nominal center of the galaxy as given by the NSA, we cull an initial 61 objects from our parent sample with an X-ray source located close to the optical galaxy center.

We then examine the optical properties of the remaining 61 galaxies in detail to determine if each is indeed a dwarf galaxy. We check the absolute magnitudes in all SDSS bands given in the NSA and redshifts from the SDSS spectra (and/or NED when available), as well as visually inspect the SDSS images. This results in the identification of many interlopers: nearby massive galaxies with erroneous mass estimates (these tend to have an obviously incorrect magnitude in an SDSS band), portions of nearby galaxies (including $\mathrm{H}$ II regions and star clusters), and faint high-redshift galaxies. Interloping massive galaxies hosting AGNs, in particular, will preferentially correlate with X-rays.

For the remaining 31 bona fide dwarf galaxies, we obtain and re-analyze the original X-ray data from the Chandra archive as described in Section 3 below. By re-analyzing the $\mathrm{X}$-ray data, and not simply using the CSC catalog products, we are able to tailor the X-ray analysis toward our specific needs. Some of the advantages (which are described in more detail in Section 3) include the following: we consider new Chandra observations that were taken after the creation of the latest CSC; we apply the latest Chandra calibration files to each image; we customize source and background photometry apertures to each individual X-ray source; we align the Chandra astrometry to the SDSS reference frame when possible, which is important for determining if an X-ray source is consistent with the optical center of each galaxy; and we are able to search for off-nuclear X-ray sources within each galaxy (the initial match to the CSC only isolates X-ray sources $5^{\prime \prime}$ from the optical nucleus).

To minimize possible contamination from diffuse X-ray emission (e.g., hot gas from star formation) and point-like soft $\mathrm{X}$-ray sources (such as $\mathrm{BH}$ and neutron star XRBs in their thermal dominant states, super-soft X-ray sources, and/or cataclysmic variables), we exclude sources that are not detected in the hard 2-7 keV band. As described below, our final sample of dwarfs with hard X-ray-selected candidate BHs consists of 19 galaxies and is given in Table 1.

\section{CHANDRA DATA REDUCTION AND PHOTOMETRY}

We obtained observations of 31 dwarf galaxies from the Chandra Data Archive, totaling a cumulative exposure time of $664 \mathrm{ks}$, and reduced the data with CIAO version 4.5 (Fruscione et al. 2006). As many of the galaxies were not targeted themselves, their positions are spread over the footprint of the detectors with varying distances from the nominal aim-points. We reprocessed the data to create new level 2 event files and new bad pixel files, taking into account if the observations were taken in FAINT or VFAINT mode. We then checked for background flares and removed time intervals in which the background rate was $>3 \sigma$ above the mean level. For each observation, we improved the Chandra astrometry by crossmatching the detected X-ray point sources to the SDSS (which has absolute astrometry accurate to $\lesssim 0$.' 1 ), and applied the resulting bore-sight corrections following Zhao et al. (2005), as follows. First, we create X-ray images, including only the $0.3-7.0 \mathrm{keV}$ range, where Chandra is best calibrated. Then, we run a wavelet detection algorithm on each activated chip using CIAO wavdetect with a sensitivity threshold corresponding to one expected spurious source per field (i.e., for a $1024 \times 1024$ pixel image, this corresponds to a wavdetect significance threshold of $\sim 10^{-6}$ ). We cross-match the list of X-ray sources with optical sources in the SDSS catalog (with $r<23 \mathrm{mag}$ ), excluding X-ray sources within three half-light radii of the target galaxy. If at least two sources were found in common, we generated a new aspect solution file for the Chandra observation. This resulted in improved astrometry for $\sim 80 \%$ of the observations, and the corrections were in the range $\sim 0$." $01-1{ }^{\prime \prime} 01$. The final astrometric solutions are accurate to between $0 . \prime 2$ and 0.5 .

After registering the Chandra images to the SDSS, we restrict further analysis to the $2-7 \mathrm{keV}$ energy band to search for hard X-ray point sources within three half-light radii of our target galaxies (as the contribution from background X-ray sources is expected to dominate beyond this; see Swartz et al. 2008). We run wavdetect on each hard image with wavelet scales of 1.0, 1.4, and 2.0 pixels, using a $4.5 \mathrm{keV}$ exposure map. As before, we adjust the wavdetect sensitivity threshold for each image so that we expect at most one spurious detection per field. This yielded a total of 60 hard $\mathrm{X}$-ray sources in 26 galaxies. For the positional accuracy of each source centroid, we estimate $95 \%$ error circles $\left(p_{\text {err }}\right)$ using the empirical formula derived by Hong et al. (2005, see their Equation (5)); this formula depends on number of hard net counts detected by wavdetect and the location of each source on the ACIS detector. The majority of the hard X-ray sources $(86 \%)$ have modest positional uncertainties $<3^{\prime \prime}$. The remaining sources are typically located toward an edge of the detector and can have uncertainties up to $\sim 10^{\prime \prime}$ in the most extreme cases.

We then perform aperture photometry on the hard X-ray images. We adopt circular apertures with radii corresponding to the $90 \%$ encircled energy fraction at $4.5 \mathrm{keV}$. The aperture sizes varied from 3 ! $^{\prime \prime}$ to 20 " 6 , depending on the off-axis position of each galaxy. In most cases, background counts were calculated from an annulus with inner and outer radii of $10^{\prime \prime}$ and $30^{\prime \prime}$ surrounding the source centroid position (masking out overlapping point-like X-ray sources when present). For sources farther from the aim-point with larger apertures, we used background annuli with inner and outer radii of $20^{\prime \prime}$ and 
$40^{\prime \prime}$. The number of counts in each background annulus are then normalized to the area of each circular source aperture, to estimate the number of expected background counts in each aperture. We use the $4.5 \mathrm{keV}$ exposure map to estimate effective exposure times at the location of each source on the detector. The net counts were then divided by 0.9 to correct for the finite aperture size.

It is possible for wavdetect to return a small number of spurious detections, so we further examine each X-ray source. Any spurious detections would be caused primarily by statistical fluctuations (i.e., the optics and detector characteristics of Chandra are well constrained and accounted for during the reduction procedure). Since we excluded time intervals subject to unusually high background levels (and limit ourselves to hard X-ray images where the background is typically low; see Table 2), random fluctuations are determined by Poisson statistics. Using the source and background apertures customized for each X-ray source above, we reassess which hard X-ray sources are detected at a statistically meaningful level. To retain a source, we require a number of counts higher than the background level (within each source aperture) at the $>95 \%$ confidence level. For observations with low sky background $(<0.5$ expected counts within the source aperture), we set the detection threshold at three counts (Gehrels 1986). For sources with large sky backgrounds, we adopt the 95\% confidence level from the Bayesian formalism of Kraft et al. (1991), where a typical threshold is 3-4 counts for cases with $0.5-1$ expected background counts within the source aperture.

Sources below the detection threshold calculated above are excluded from further analysis. This resulted in 17 of the 60 detected sources being excluded, and the remaining 43 detected $\mathrm{X}$-ray sources are found in a total of 19 galaxies. The coordinates of each X-ray source are listed in Table 2 in order of increasing distance from the nominal optical position of their host galaxy, and we also note in Table 2 which sources are also included in the CSC. Given our adopted 95\% detection confidence limit, we expect that $5 \%$ of the sources in Table 2 $(\sim 2 / 43)$ could still be false positives due to random statistical fluctuations. Although 5\% is likely an upper limit, given that many have counts well above the $95 \%$ confidence limit, all were deemed detections by wavdetect, and many X-ray sources (30/43) are also included in the CSC.

Next, we estimate unabsorbed $2-10 \mathrm{keV}$ fluxes using version 4.6b of the Portable, Interactive Multi-Mission Simulator ${ }^{5}$, accounting for Galactic foreground absorption toward each galaxy following Dickey \& Lockman (1990), and assuming a power-law spectrum with photon index $\Gamma=1.8$, typical of accreting massive $\mathrm{BHs}$ at moderate Eddington ratios (Ho 2009), hard-state XRBs, as well as many ULXs (Swartz et al. 2008; Feng \& Soria 2011). Net count rates, unabsorbed fluxes, and luminosities are given in Table 2 . These values may be considered lower limits as there may be additional absorption intrinsic to the sources. All error bars are quoted at the $90 \%$ confidence level, unless stated otherwise.

Finally, we assess the expected number of foreground/ background sources within three half-light radii of each galaxy. Following Plotkin et al. (2014), we use the published X-ray source counts from the resolved cosmic X-ray background as given by Moretti et al. (2003). Starting with the hard (2-10 keV) X-ray flux $S_{\text {dim }}$, which corresponds to the dimmest $\mathrm{X}$-ray source in each field, we adopt the expression for the cumulative X-ray flux distribution given in Equation (2) by Moretti et al. (2003) to calculate $N(>S)$, i.e., the expected number of X-ray sources per square degree having an X-ray flux brighter than $S$. Finally, for each galaxy field, $N(>S)$ is multiplied by the area enclosed by three half-light radii. With the exception of one nearby galaxy with a moderately deep exposure (ID 11, NGC 4214; $N_{\mathrm{bk}}=4.8$ ), the expected number of background sources, $N_{\mathrm{bk}}$, varies between 0.00025 and 0.74

Table 1

Dwarf Galaxy Sample

\begin{tabular}{|c|c|c|c|c|c|c|c|c|}
\hline $\begin{array}{l}\mathrm{ID} \\
(1)\end{array}$ & $\begin{array}{c}\text { NSAID } \\
(2)\end{array}$ & $\begin{array}{c}\text { SDSS Name } \\
\text { (3) }\end{array}$ & $\begin{array}{c}\text { Other Name } \\
\text { (4) }\end{array}$ & $\begin{array}{c}\log M_{\star} \\
(5)\end{array}$ & $\begin{array}{c}z \\
(6)\end{array}$ & $\begin{array}{l}N_{\mathrm{H}} \\
(7) \\
\end{array}$ & $\begin{array}{r}M_{g} \\
(8) \\
\end{array}$ & $\begin{array}{l}r_{50} \\
(9) \\
\end{array}$ \\
\hline 1 & 130302 & $\mathrm{~J} 0144446.40+170634.0$ & MRK 0361 & 9.230 & 0.027 & 5.03 & -20.0 & 2.53 \\
\hline 2 & 131489 & $\mathrm{~J} 021403.59+275238.0$ & NGC 0855 & 9.054 & 0.002 & 6.41 & -17.3 & 16.16 \\
\hline 4 & 135806 & J091721.89+415437.9 & UGC 04904 & 9.068 & 0.006 & 1.03 & -17.7 & 12.41 \\
\hline 5 & 135954 & $\mathrm{~J} 093401.99+551427.9$ & I Zw 18, MRK 0116 & 6.599 & 0.003 & 1.99 & -14.9 & 3.09 \\
\hline 6 & 34462 & $\mathrm{~J} 103231.89+542403.5$ & UGC 05720 & 8.934 & 0.005 & 0.90 & -19.2 & 4.51 \\
\hline 9 & 3264 & J115237.19-022809.9 & UGC 06850 & 7.812 & 0.003 & 2.25 & -16.8 & 6.39 \\
\hline 10 & 41753 & $\mathrm{~J} 121326.02+543631.8$ & $\ldots$ & 7.607 & 0.008 & 1.43 & -15.0 & 5.16 \\
\hline 11 & 140999 & $\mathrm{~J} 121539.20+361937.0$ & NGC 4214 & 9.071 & 0.001 & 1.49 & -19.0 & 43.08 \\
\hline 12 & 30830 & $\mathrm{~J} 121923.08+054741.4$ & VCC 0344 & 9.417 & 0.007 & 1.58 & -17.3 & 2.44 \\
\hline 13 & 117784 & $\mathrm{~J} 122111.29+173819.1$ & $\ldots$ & 8.850 & 0.007 & 2.68 & -17.7 & 6.84 \\
\hline 14 & 161603 & J122219.80+300347.9 & $\ldots$ & 8.565 & 0.002 & 1.73 & -15.6 & 15.78 \\
\hline 19 & 92811 & $\mathrm{~J} 132501.28+362613.7$ & NGC 5143 & 9.342 & 0.019 & 0.92 & -18.9 & 6.11 \\
\hline
\end{tabular}

Note. Column 1: identification number assigned in this paper. Column 2: NASA-Sloan Atlas identification number. Column 3: SDSS name. Column 4: alternative names. Column 5: $\log$ galaxy stellar mass in units of $M_{\odot}$. Column 6: heliocentric redshift. Column 7: neutral hydrogen column density in units of $10^{20} \mathrm{~cm}^{-2}$. Column 8: absolute $g$-band magnitude corrected for foreground Galactic exctinction. Column 9: Petrosian 50\% light radius in units of arcseconds. With the exception of columns 4 and 7, all values are from the NSA and assume $h=0.73$. 
Table 2

Hard X-ray Sources

\begin{tabular}{|c|c|c|c|c|c|c|c|c|c|c|}
\hline $\begin{array}{l}\text { ID } \\
(1)\end{array}$ & $\begin{array}{l}\text { R.A. } \\
(2)\end{array}$ & $\begin{array}{c}\text { Decl. } \\
(3)\end{array}$ & $\begin{array}{l}P_{\text {err }} \\
(4)\end{array}$ & $\begin{array}{c}\text { Net Counts } \\
(5)\end{array}$ & $\begin{array}{l}N_{\text {bg }} \\
(6)\end{array}$ & $\begin{array}{c}\text { Exposure Time } \\
\text { (7) }\end{array}$ & $\begin{array}{c}F_{2-10 \mathrm{keV}} \\
\quad(8)\end{array}$ & $\begin{array}{c}\log L_{2-10 \mathrm{keV}} \\
(9)\end{array}$ & $\begin{array}{c}d \\
(10)\end{array}$ & $\begin{array}{c}\text { CXO ID } \\
(11)\end{array}$ \\
\hline 1 & 26.193495 & 17.109842 & 3.20 & $3.11_{-2.37}^{+3.63}$ & 0.2 & 13.70 & 4.93 & 39.9 & 1.42 & J014446.4+170634 \\
\hline 2 & 33.516998 & 27.877712 & 1.06 & $3.31_{-2.39}^{+3.62}$ & 0.1 & 1.54 & 46.95 & 38.7 & 7.88 & J021404.0+275239 \\
\hline 3 & 75.433619 & -4.288618 & 0.40 & $21.77_{-7.07}^{+9.37}$ & 0.5 & 33.77 & 12.80 & 39.7 & 0.25 & J050144.0-041718 \\
\hline$\ldots$ & 75.433663 & -4.289359 & 0.40 & $23.99_{-7.45}^{+9.75}$ & 0.5 & $\ldots$ & 14.11 & 39.7 & 2.47 & J050144.0-041721 \\
\hline$\ldots$ & 75.431942 & -4.291328 & 0.41 & $18.43_{-6.45}^{+8.77}$ & 0.5 & $\cdots$ & 10.84 & 39.6 & 11.39 & J050143.6-041728 \\
\hline 4 & 139.337673 & 41.911788 & 0.33 & $79.47_{-14.07}^{+16.30}$ & 0.5 & 51.64 & 33.25 & 39.4 & 7.61 & J091721.0+415442 \\
\hline$\ldots$ & 139.343966 & 41.909299 & 0.34 & $56.13_{-11.73}^{+13.98}$ & 0.5 & $\ldots$ & 23.49 & 39.3 & 11.48 & J091722.5+415433 \\
\hline$\ldots$ & 139.336623 & 41.909045 & 0.31 & $190.58_{-22.13}^{+24.32}$ & 0.5 & $\ldots$ & 79.75 & 39.8 & 11.49 & J091720.8+415432 \\
\hline$\cdots$ & 139.331982 & 41.910328 & 0.79 & $2.80_{-2.34}^{+3.64}$ & 0.5 & $\cdots$ & 1.17 & 38.0 & 22.06 & $\cdots$ \\
\hline$\cdots$ & 139.346643 & 41.906837 & 0.41 & $15.02_{-5.76}^{+8.10}$ & 0.5 & $\ldots$ & 6.29 & 38.7 & 22.45 & J091723.2+415424 \\
\hline$\cdots$ & 139.345338 & 41.901709 & 0.59 & $3.91_{-2.88}^{+3.96}$ & 0.5 & $\ldots$ & 1.64 & 38.1 & 35.55 & $\cdots$ \\
\hline 5 & 143.508191 & 55.241235 & 0.57 & $5.51_{-3.42}^{+4.17}$ & 0.1 & 1.28 & 94.33 & 39.3 & 0.50 & J093401.9+551428 \\
\hline 6 & 158.133570 & 54.400612 & 0.40 & $17.54_{-6.28}^{+8.60}$ & 0.2 & 16.38 & 21.08 & 39.2 & 0.81 & $\mathrm{~J} 103232.0+542402$ \\
\hline$\ldots$ & 158.131360 & 54.403201 & 0.51 & $6.43_{-3.86}^{+4.42}$ & 0.2 & $\ldots$ & 7.73 & 38.8 & 9.79 & $\mathrm{~J} 103231.5+542411$ \\
\hline 7 & 158.542998 & 58.062962 & 9.73 & $34.70_{-9.09}^{+11.36}$ & 2.0 & 9.47 & 89.58 & 40.1 & 3.02 & $\mathrm{~J} 103410.1+580346$ \\
\hline$\ldots$ & 158.539409 & 58.063876 & 9.85 & $23.57_{-7.38}^{+9.68}$ & 2.0 & $\ldots$ & 60.84 & 39.9 & 5.63 & $\ldots$ \\
\hline 8 & 162.836691 & 32.765375 & 0.51 & $15.49_{-5.86}^{+8.20}$ & 0.1 & 1.73 & 193.39 & 39.6 & 3.43 & $\mathrm{~J} 105120.8+324555$ \\
\hline 9 & 178.155613 & -2.468589 & 0.53 & $14.39_{-5.62}^{+7.97}$ & 0.1 & 5.06 & 61.57 & 39.3 & 3.81 & J115237.3-022807 \\
\hline 10 & 183.360607 & 54.609860 & 8.95 & $91.51_{-15.15}^{+17.37}$ & 6.3 & 29.61 & 66.81 & 40.0 & 6.35 & $\mathrm{~J} 121326.1+543634$ \\
\hline 11 & 183.920218 & 36.327396 & 0.65 & $8.55_{-4.69}^{+4.80}$ & 0.3 & 26.03 & 7.47 & 37.7 & 17.50 & $\mathrm{~J} 121540.8+361939$ \\
\hline$\cdots$ & 183.908986 & 36.328876 & 0.41 & $45.28_{-10.47}^{+12.73}$ & 0.3 & $\ldots$ & 39.57 & 38.4 & 17.85 & $\mathrm{~J} 121538.1+361944$ \\
\hline$\cdots$ & 183.919917 & 36.323634 & 0.76 & $5.38_{-3.41}^{+4.18}$ & 0.2 & $\ldots$ & 4.70 & 37.5 & 19.07 & $\cdots$ \\
\hline$\cdots$ & 183.909394 & 36.322301 & 0.33 & $235.24_{-24.65}^{+26.84}$ & 0.3 & $\cdots$ & 205.57 & 39.1 & 20.50 & $\mathrm{~J} 121538.2+361921$ \\
\hline$\ldots$ & 183.921454 & 36.315876 & 0.62 & $6.41_{-3.86}^{+4.42}$ & 0.3 & $\ldots$ & 5.60 & 37.6 & 43.16 & $\ldots$ \\
\hline$\cdots$ & 183.900242 & 36.312919 & 0.65 & $5.32_{-3.40}^{+4.19}$ & 0.2 & $\cdots$ & 4.65 & 37.5 & 63.39 & $\cdots$ \\
\hline$\ldots$ & 183.936452 & 36.312645 & 0.52 & $10.86_{-5.46}^{+5.11}$ & 0.3 & $\ldots$ & 9.49 & 37.8 & 81.08 & $\mathrm{~J} 121544.7+361846$ \\
\hline$\cdots$ & 183.922478 & 36.353828 & 0.70 & $21.89_{-7.09}^{+9.39}$ & 0.3 & $\cdots$ & 19.13 & 38.1 & 101.57 & J121541.4+362114 \\
\hline$\ldots$ & 183.935980 & 36.302550 & 0.80 & $3.10_{-2.37}^{+3.63}$ & 0.2 & $\ldots$ & 2.71 & 37.3 & 106.38 & $\ldots$ \\
\hline 12 & 184.846310 & 5.794871 & 0.80 & $6.41_{-4.17}^{+4.73}$ & 1.4 & 62.97 & 2.00 & 38.3 & 0.39 & $\mathrm{~J} 121923.1+054741$ \\
\hline$\ldots$ & 184.847546 & 5.796062 & 2.00 & $4.20_{-3.28}^{+4.27}$ & 1.4 & $\ldots$ & 1.32 & 38.1 & 6.50 & $\ldots$ \\
\hline$\cdots$ & 184.845120 & 5.793374 & 1.31 & $3.04_{-2.75}^{+3.90}$ & 1.4 & $\cdots$ & 0.95 & 38.0 & 6.57 & J121922.8+054735 \\
\hline 13 & 185.296810 & 17.636862 & 11.25 & $14.30_{-5.60}^{+7.95}$ & 1.3 & 4.07 & 69.98 & 39.9 & 6.61 & $\mathrm{~J} 122111.0+173817$ \\
\hline 14 & 185.580669 & 30.061350 & 4.76 & $3.33_{-2.39}^{+3.62}$ & 0.1 & 4.10 & 16.16 & 38.6 & 3.65 & $\mathrm{~J} 122219.3+300340$ \\
\hline 15 & 186.453622 & 33.546937 & 0.30 & $876.17_{-48.12}^{+50.28}$ & 0.5 & 10.58 & 1882.84 & 40.1 & 0.25 & $\mathrm{~J} 122548.8+333248$ \\
\hline$\cdots$ & 186.452117 & 33.550481 & 0.77 & $2.83_{-2.34}^{+3.64}$ & 0.5 & $\cdots$ & 6.09 & 37.7 & 13.77 & $\cdots$ \\
\hline 16 & 191.321862 & 27.125506 & 0.34 & $52.00_{-11.27}^{+13.52}$ & 0.2 & 15.82 & 68.10 & 39.4 & 0.40 & $\mathrm{~J} 124517.2+270731$ \\
\hline$\cdots$ & 191.320909 & 27.126584 & 0.40 & $18.66_{-6.49}^{+8.82}$ & 0.2 & $\cdots$ & 24.44 & 39.0 & 4.82 & $\cdots$ \\
\hline$\cdots$ & 191.320436 & 27.126193 & 0.39 & $20.89_{-6.91}^{+9.22}$ & 0.2 & $\ldots$ & 27.35 & 39.0 & 5.24 & $\ldots$ \\
\hline 17 & 192.298020 & 3.389506 & 0.55 & $5.43_{-3.41}^{+4.18}$ & 0.1 & 9.00 & 1.30 & 38.3 & 3.05 & $\mathrm{~J} 124911.5+032322$ \\
\hline$\cdots$ & 192.291310 & 3.379165 & 0.73 & $3.22_{-2.38}^{+3.63}$ & 0.1 & $\cdots$ & 7.71 & 38.1 & 42.54 & $\cdots$ \\
\hline 18 & 192.481030 & 5.319315 & 0.98 & $4.42_{-2.93}^{+3.92}$ & 0.1 & 4.40 & 2.23 & 38.5 & 46.22 & $\cdots$ \\
\hline$\cdots$ & 192.473880 & 5.311602 & 0.55 & $7.72_{-4.30}^{+4.60}$ & 0.1 & $\ldots$ & 3.90 & 38.8 & 61.98 & $\mathrm{~J} 124953.7+051841$ \\
\hline 19 & 201.255760 & 36.437561 & 1.40 & $3.30_{-2.39}^{+3.62}$ & 0.1 & 4.61 & 15.87 & 40.1 & 2.21 & $\mathrm{~J} 132501.3+362615$ \\
\hline
\end{tabular}

Note. Column 1: galaxy ID (see Table 1). Column 2: right ascension of hard X-ray source in units of degrees. Column 3: declination of hard X-ray source in units of degrees. Column 4: 95\% positional uncertainty in arcseconds. The values do not include the 0."2-0."5 uncertainties from the astrometric correction. Column 5: net counts in the energy range 2-7 keV, after applying a 90\% aperture correction to the net counts in each source extraction circle. Error bars represent $90 \%$ confidence intervals. Column 6: number of background counts expected within each source extraction circle (after applying a 90\% aperture correction). Column 7: effective exposure time in kiloseconds at each source position on the detector, determined from $4.5 \mathrm{keV}$ exposure maps. Column 8: 2-10 keV flux in units of $10^{-15} \mathrm{erg} \mathrm{s}^{-1} \mathrm{~cm}^{-2}$ corrected for Galactic absorption. Column 9: $\log 2-10 \mathrm{keV}$ luminosity in units of erg s ${ }^{-1}$ corrected for Galactic absorption. Column 10: distance from optical center of the galaxy in units of arcseconds. Column 11: name of source in CSC catalog, if present. Sources are listed in increasing distance from the center of the galaxy, and sources with positions consistent with the optical nucleus are highlighted in boldface (see Section 4.1).

with a median of 0.016. Across the sample, we expect a total of 1.4 background contaminants (excluding ID 11).

5 See http://heasarc.gsfc.nasa.gov/Tools/w3 pimms.html.

\section{ANALYSIS AND RESULTS}

We detect a total of 43 point-like hard X-ray sources in 19 dwarf galaxies (Figure 1). Approximately $42 \%$ of our sample 

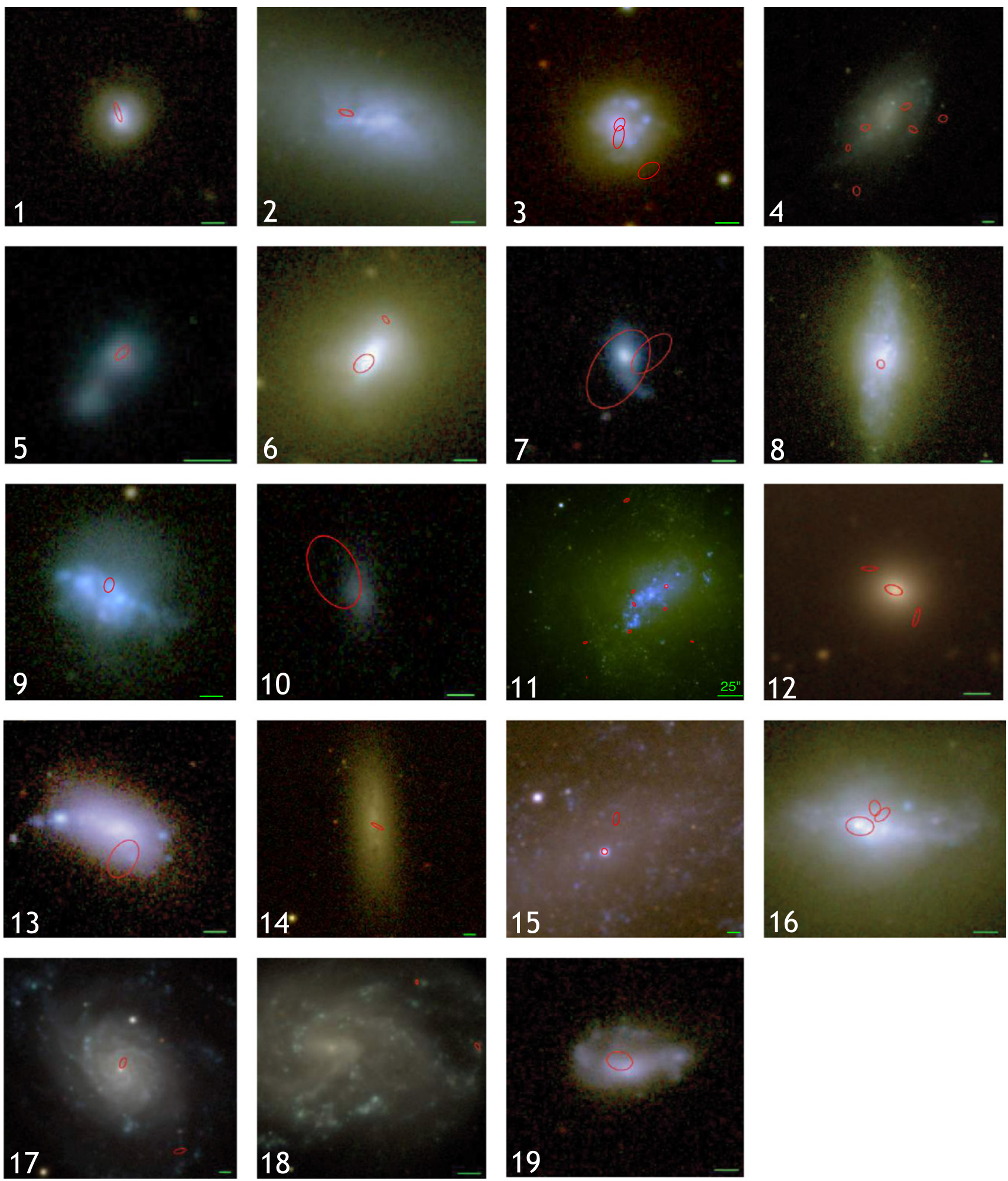

Figure 1. SDSS images of our dwarf galaxy sample. Red ellipses indicate the positions of hard X-ray sources (from wavdetect). Scale bars are 5" in length, except for ID 11 , which has a $25^{\prime \prime}$ scale bar.

of galaxies (8/19) have X-ray detections reported here for the first time-we searched the NED databases to identify any X-ray sources that are already known to be background/ foreground objects or otherwise reported in the literature. Notes on individual objects are in the Appendix.

\subsection{Spatial Distribution of X-ray Sources and Candidate Nuclear Sources}

In Figure 2, we show the distribution of the locations of the hard X-ray sources within each galaxy normalized by the halflight radius. Roughly half of the X-ray sources (23/43) fall 


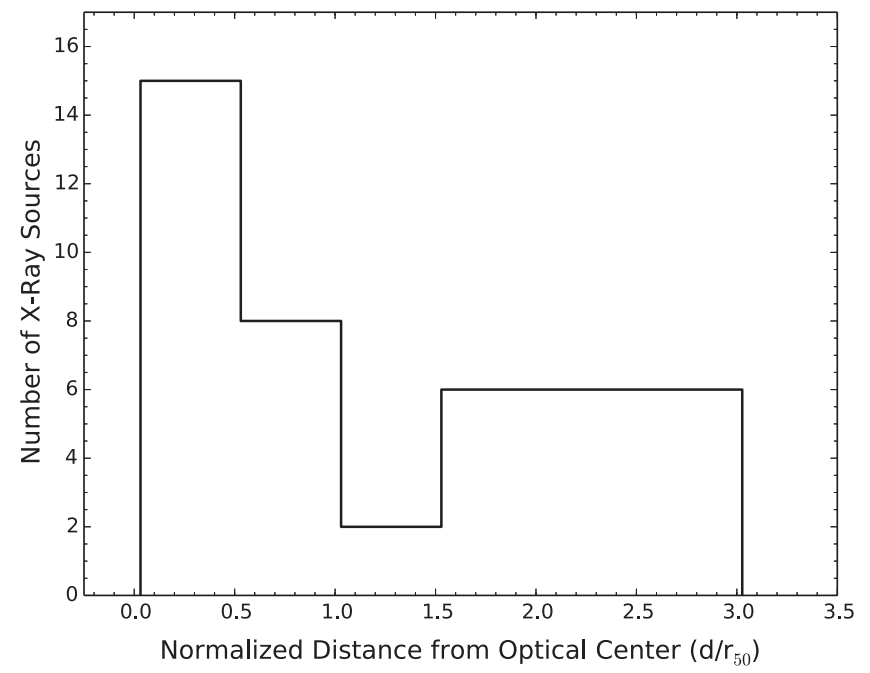

Figure 2. Distribution of the distances of hard X-ray sources from the optical center of their host galaxy, normalized by the half-light radius.

within one half-light radius of the optical galaxy center (as given by the NSA). Such a distribution is expected for the (offnuclear) luminous XRB population (e.g., Swartz et al. 2011). It is possible that some of the X-ray detections close to the optical center could be AGNs and not XRBs. However, since the positional accuracy degrades for sources falling toward the edge of the chip, it is difficult to apply uniform criteria across the entire sample to identify likely nuclear X-ray sources. Instead, we determine which X-ray sources have 95\% error circles that overlap with the optical center (we add $\sim 0$.'5 uncertainty from the astrometric correction to the $p_{\text {err }}$ estimates), and we find that $11 \mathrm{X}$-ray sources (in 10 galaxies) are consistent with the nucleus of the host galaxy. We caution that the optical center of a galaxy may not be well defined for irregular galaxies or those with star-forming clumps.

We stress that we consider 11 "nuclear" X-ray sources to be a generous upper limit, as many of these sources are consistent with the optical center primarily because the error circles on their positions are large (mostly due to galaxies falling toward an edge of the detector). For example, five X-ray sources (in galaxy IDs $7,10,13,14)$ have positional uncertainties $p_{\text {err }} \gtrsim 5^{\prime \prime}$ (with X-ray positions $\sim 3^{\prime \prime}-7^{\prime \prime}$ from the optical center), and two of these sources fall within the same galaxy (ID 7). These X-ray sources could very likely be off-nuclear XRBs. One previously unidentified X-ray source (in galaxy ID 1; see Appendix) lies 1". $4 \pm 3$ ". 2 from the optical center and could be an intriguing nuclear source. The remaining sources (IDs 3, 5, 12, 15, 16) have much more accurate positions $\left(p_{\text {err }}<1^{\prime \prime}\right)$ and lie $<0$ ".5 from the optical center. Two of these are newlyidentified objects, and three are previously identified X-ray sources, including the low-mass Seyfert galaxy NGC 4395 (see Appendix).

\subsection{Host Galaxy Properties}

We obtained galaxy stellar masses and half-light radii from the NSA. The stellar masses span a range of $M_{\star} \sim 10^{7}-10^{9.5} M_{\odot}$, with a median of $\sim 10^{9} M_{\odot}$. The galaxies in our sample are physically small in addition to being low mass, with half-light radii $\lesssim 2 \mathrm{kpc}$. The galaxies span a range of optical colors, and our sample includes spheroids, disks, and irregular systems (see Figure 1).
Table 3

Expected Galaxy-wide Luminosity from X-ray Binaries

\begin{tabular}{|c|c|c|c|c|c|c|}
\hline $\begin{array}{l}\text { ID } \\
(1)\end{array}$ & $\begin{array}{l}\text { FUV } \\
(2)\end{array}$ & $\begin{array}{c}L(\mathrm{FUV})_{\mathrm{obs}} \\
(3)\end{array}$ & $\begin{array}{l}W_{22} \\
(4)\end{array}$ & $\begin{array}{c}L(25 \mu \mathrm{m}) \\
(5)\end{array}$ & $\begin{array}{c}\log \text { SFR } \\
(6)\end{array}$ & $\begin{array}{c}\log L \\
\text { XRB } \\
2-10 \mathrm{keV} \\
\quad(7)\end{array}$ \\
\hline 1 & 16.44 & 43.45 & 5.01 & 43.17 & 0.58 & 39.80 \\
\hline 2 & 15.20 & 41.67 & 5.39 & 40.74 & -1.52 & 38.18 \\
\hline 3 & 15.42 & 43.24 & 6.01 & 42.15 & 0.01 & 39.27 \\
\hline 4 & 16.54 & 42.04 & 7.58 & 40.76 & -1.23 & 38.30 \\
\hline 5 & 15.79 & 41.64 & 7.84 & 39.97 & -1.67 & 37.54 \\
\hline 6 & 14.24 & 42.83 & 2.53 & 42.66 & 0.04 & 39.26 \\
\hline 7 & 16.42 & 42.34 & 7.45 & 41.07 & -0.93 & 38.29 \\
\hline 8 & 15.10 & 41.77 & 5.53 & 40.75 & -1.44 & 38.10 \\
\hline 9 & 15.05 & 42.22 & 4.68 & 41.52 & -0.88 & 38.34 \\
\hline 10 & 19.57 & 41.13 & $>9.28$ & $<40.40$ & -2.22 & 37.13 \\
\hline 11 & 11.57 & 42.51 & 2.62 & 41.23 & -0.76 & 38.59 \\
\hline 12 & 21.14 & 40.37 & $>8.86$ & $<40.42$ & -2.98 & 38.38 \\
\hline 13 & 16.31 & 42.32 & $>8.07$ & $<40.77$ & -1.03 & 38.34 \\
\hline 14 & 19.73 & 40.00 & 8.84 & 39.50 & -3.00 & 37.54 \\
\hline 15 & 13.65 & 41.75 & 5.52 & 40.15 & -1.56 & 38.20 \\
\hline 16 & 13.48 & 42.89 & 3.96 & 41.84 & -0.33 & 38.92 \\
\hline 17 & 14.73 & 42.03 & 4.54 & 41.25 & -1.10 & 38.46 \\
\hline 18 & 14.22 & 42.14 & 5.91 & 40.61 & -1.16 & 38.41 \\
\hline 19 & 17.37 & 42.79 & 8.12 & 41.64 & -0.45 & 38.89 \\
\hline
\end{tabular}

Note. Column 1: galaxy ID (see Table 1). Column 2: GALEX far-UV AB magnitude. Column 3: observed far-UV luminosity in units of $\mathrm{erg} \mathrm{s}^{-1}$. Column 4: WISE $22 \mu \mathrm{m}$ Vega magnitude. Column 5: $25 \mu \mathrm{m}$ luminosity in units of $\mathrm{erg} \mathrm{s}^{-1}$, using $22 \mu \mathrm{m}$ flux density (in Jy) as a proxy for $25 \mu \mathrm{m}$ flux density (Jarrett et al. 2013). Column 6: $\log$ SFR in units of $M_{\odot} \mathrm{yr}^{-1}$. Column 7: total $2-10 \mathrm{keV}$ luminosity expected from low-mass and high-mass XRBs. Units are in $\mathrm{erg} \mathrm{s}^{-1}$.

We estimate star formation rates (SFRs) of the host galaxies using a combination of far-UV (FUV) data from GALEX and $22 \mu \mathrm{m}$ data from the Wide-field Infrared Survey Explorer (WISE; Wright et al. 2010). GALEX FUV magnitudes were obtained from the NSA, and infrared magnitudes were obtained from the WISE All-Sky Source Catalog (see Table 3). Following Kennicutt \& Evans (2012) and Hao et al. (2011), we estimate dust-corrected SFRs as $\log$ SFR $=\log$ $L(\mathrm{FUV})_{\text {corr }}-43.35, \quad$ where $\quad L(\mathrm{FUV})_{\text {corr }}=L(\mathrm{FUV})_{\mathrm{obs}}$ $+3.89 L(25 \mu \mathrm{m})$ and the luminosities are in units of $\mathrm{erg} \mathrm{s}^{-1}$. While the Hao et al. (2011) relation employs $25 \mu \mathrm{m}$ luminosities from the Infrared Astronomical Satellite (IRAS), the vast majority of our sample is not detected by IRAS at $25 \mu \mathrm{m}$. WISE is sufficiently more sensitive, and all but three of our sample galaxies have $22 \mu \mathrm{m}$ detections. Given this, and the fact that we expect the flux density ratio between 22 and $25 \mu \mathrm{m}$ (in Jy) to be $\sim 1$ for both late-type and early-type galaxies (Jarrett et al. 2013), we use $22 \mu \mathrm{m}$ flux densities as a proxy for $25 \mu \mathrm{m}$ flux densities. For the three galaxies not detected at $22 \mu \mathrm{m}$ in WISE, we derive SFRs using the observed (uncorrected) FUV luminosities. The derived SFRs are given in Table 3 . We plot specific SFRs $\left(\mathrm{sSFR}=\mathrm{SFR} / M_{\star}\right)$ versus $r-i$ optical color for our sample of dwarf galaxies in Figure 3. As expected, bluer (redder) galaxies have higher (lower) sSFRs.

\subsection{Expected Contribution from $X$-ray Binaries}

The luminosities of individual hard X-ray sources in our sample are in the range $L_{2-10 \mathrm{keV}} \sim 10^{37}-10^{40} \mathrm{erg} \mathrm{s}^{-1}$. While we are primarily interested in finding candidate massive $\mathrm{BHs}$, 




Figure 3. Specific star formation rate vs. $r-i$ optical color for the 19 galaxies in our sample. The color bar indicates $r-i$ color.

these luminosities could also be attained by stellar-mass BHs (or neutron stars) in XRBs with hard X-ray spectra (see, e.g., McClintock \& Remillard 2006). The X-ray luminosity function of XRBs in external galaxies (Fabbiano 2006) is known to scale with SFR for late-type galaxies (Grimm et al. 2002, 2003; Gilfanov et al. 2004; Mineo et al. 2012) and with total stellar mass for early-type galaxies (Gilfanov 2004; Humphrey \& Buote 2008; Lehmer et al. 2010, 2014), the former being dominated by young, bright high-mass XRBs and the latter by longer-lived low-mass XRBs.

Given our heterogeneous sample of galaxies, we estimate the expected collective luminosity from XRBs in a given galaxy using stellar masses and SFRs from Section 4.2 and the relation in Lehmer et al. (2010), which accounts for both high-mass and low-mass XRBs: $L_{2-10 \mathrm{keV}}^{\mathrm{XRB}}=\alpha M_{\star}+\beta$ SFR, where $\alpha=(9.05 \pm 0.37) \times 10^{28} \mathrm{erg} \mathrm{s}^{-1} M_{\odot}^{-1}$ and $\beta=$ $(1.62 \pm 0.22) \times 10^{39} \mathrm{erg} \mathrm{s}^{-1}\left(M_{\odot} \mathrm{yr}^{-1}\right)^{-1}$. While the Lehmer et al. (2010) relation was derived from more massive and more luminous galaxies, the range of sSFRs in that work is comparable to our sample $\left(\mathrm{SFR} / M_{\star} \sim 10^{-12}-10^{-8.5} \mathrm{yr}^{-1}\right)$. In Figure 4, we plot the total observed hard X-ray luminosity versus the expected galaxy-wide luminosity from XRBs.

We caution that there are large uncertainties associated with the expected total luminosities from XRBs. The Lehmer et al. (2010) relation itself has a scatter of $\sim 0.34$ dex. There are also large uncertainties in the stellar masses and SFRs. Uncertainties in the stellar masses are expected to be $\sim 0.3$ dex (Conroy et al. 2009) and the scatter in the Hao et al. (2011) relation is 0.13 dex. Stochastic effects are also a concern for galaxies with low stellar masses and/or low SFRs since a small number of luminous XRBs can dominate the total X-ray luminosity. The $1 \sigma$ error bars $( \pm 0.4 \mathrm{dex})$ in Figure 4 account for systematic uncertainties in stellar masses and SFRs, scatter in the Lehmer et al. (2010) relation, as well as errors on $\alpha$ and $\beta$ in Lehmer et al. (2010).

\subsection{Galaxies with Enhanced X-ray Emission}

Eight galaxies in our sample $(42 \%)$ exhibit excess hard $\mathrm{X}$-ray emission relative to the expected contribution from XRBs (Figure 4). These galaxies have observed hard X-ray emission $>3 \sigma$ (or more than $1.2 \mathrm{dex}$ ) higher than expected given their stellar masses and SFRs. The X-ray sources responsible for the excess emission in five of these cases also have positions consistent with the optical centers of the host galaxies as described in Section 4.1 (IDs 5, 7, 10, 13, 15). ID 15 is the well-studied dwarf Seyfert galaxy NGC 4395 (Filippenko \& Sargent 1989), and ID 5 is the extremely metal-poor galaxy I Zw 18 with a well-studied luminous X-ray source (see Appendix). To the best of our knowledge, the $\mathrm{X}$-ray sources in galaxies 7, 10, and 13 are reported here for the first time. However, we note that these galaxies have somewhat irregular morphologies without clearly defined nuclei, and the X-ray positions are not well constrained. An additional three galaxies (IDs 4, 8, 19) exhibit enhanced X-ray emission from non-nuclear sources (see Figure 4).

\subsection{X-ray Detection Limits}

Given the heterogeneous nature of our galaxy sample, each galaxy has a different luminosity threshold to which an X-ray source could be detected. To estimate the minimum detectable luminosity, we assume that three hard X-ray photons $(2-7 \mathrm{keV})$ must be detected within the source aperture (corresponding to a $95 \%$ confidence limit if there is no contribution from the background; Gehrels 1986). More photons would be required if there is significant contribution from the local background, so we consider the assumption for three photons to provide a robust lower limit. Then, assuming the effective exposure time for each galaxy, the Galactic foreground absorption, the distance to each galaxy, and a power-law spectrum with photon index $\Gamma=1.8$, we calculate the minimum $2-10 \mathrm{keV}$ luminosity $\left(L_{\min }\right)$ for which we could detect a hard $\mathrm{X}$-ray source within each galaxy. The sensitivity limits range from $5 \times 10^{36}$ to $1 \times 10^{40} \mathrm{erg} \mathrm{s}^{-1}$, with a median value of $2.3 \times 10^{38} \mathrm{erg} \mathrm{s}^{-1}$. The distribution of observed X-ray luminosities is largely shaped by these detection limits (see Figure 4).

\section{SUMMARY AND DISCUSSION}

We have constructed a sample of 43 hard X-ray selected $\mathrm{BH}$ candidates in 19 nearby dwarf galaxies with stellar masses $M_{\star} \lesssim 3 \times 10^{9} M_{\odot}$. Eight of the galaxies in our sample have $\mathrm{X}$-ray detections reported here for the first time. After combining the total hard X-ray flux from all point sources within each galaxy, nearly all of the galaxies have cumulative hard X-ray luminosities that are higher than expected from the combination of low-mass and high-mass XRBs (given each galaxy's stellar mass and SFR). This high level of X-ray emission is consistent with expectations, given the X-rayselected nature of our galaxy sample and the sensitivity limit of each Chandra observation. For the majority of galaxies, we are typically capable of detecting a hard X-ray source with $L_{2-10 \mathrm{keV}} \gtrsim 10^{38} \mathrm{erg} \mathrm{s}^{-1}$. Therefore, our study is generally only sensitive to the luminous tail of the XRB population and more massive accreting BHs. 



Figure 4. Left: total observed $2-10 \mathrm{keV}$ luminosity vs. expected total luminosity from low-mass and high-mass XRBs. Points are color-coded by $r-i$ color. The solid line shows the one-to-one relation, and the dashed line shows our median X-ray sensitivity limit. The $1 \sigma$ error bar accounts for systematic uncertainties in stellar masses and SFRs, scatter in the Lehmer et al. (2010) relation, and errors on $\alpha$ and $\beta$ in the Lehmer et al. (2010) relation. Galaxies with statistically significant excess hard X-ray emission $(>3 \sigma$, or $1.2 \mathrm{dex})$ are marked with an additional circle. Right: same as left panel, except with X-ray emission removed from point sources consistent with the optical center (see Section 4.1).

Interestingly, even after considering the above bias, we find that a substantial fraction of the galaxies in our sample (42\%) exhibit statistically meaningful enhanced hard X-ray emission, the majority of which have X-ray sources consistent with the optical nucleus. The dwarf Seyfert 1 galaxy NGC 4395 (Filippenko \& Sargent 1989) is included in this sub-sample, demonstrating that searching for enhanced (cumulative) hard $\mathrm{X}$-ray emission can indeed be a useful diagnostic for identifying AGNs in dwarf galaxies. However, enhanced hard $\mathrm{X}$-ray emission is neither a necessary nor sufficient requirement for an accreting massive $\mathrm{BH}$. Enhanced galaxy-wide X-ray emission from off-nuclear X-ray sources (thought to be luminous XRBs) has been observed in other studies, especially in low-metallicity dwarfs, where the enhancement is up to a factor of 10 larger than for $\sim$ solar-metallicity galaxies (Prestwich et al. 2013; Brorby et al. 2014).

Follow-up X-ray observations, as well as observations at other wavelengths, can help distinguish between stellar-mass XRBs and more massive BHs in our sample. High-resolution radio observations offer a promising way forward since the ratio of compact radio to hard $\mathrm{X}$-ray emission is significantly higher for accreting massive BHs compared to stellar mass BHs (e.g., Merloni et al. 2003). The combination of radio and X-ray observations has so far revealed candidate massive BHs in the dwarf galaxies Henize 2-10 (Reines et al. 2011; Reines \& Deller 2012) and Mrk 709 (Reines et al. 2014). Eleven galaxies in our sample are detected in either the FIRST $^{6}$ or NVSS surveys; however, the angular resolution is not sufficient to associate radio emission with any particular X-ray source. MidIR diagnostics (e.g., with WISE) have been used to select luminous AGNs with high confidence (e.g., Stern et al. 2012; Assef et al. 2013); however using mid-IR colors to identify

\footnotetext{
6 The VLA FIRST Survey: Faint Images of the Radio Sky at Twenty $\mathrm{cm}$.

7 The NRAO VLA Sky Survey, Condon et al. (1998).
}

AGNs in dwarf galaxies is not straightforward. For example, star-forming dwarf galaxies can have mid-IR colors overlapping those of WISE-selected AGNs (Jarrett et al. 2011; Izotov et al. 2014). Regardless of the masses of the BHs in our sample, our results have important implications for the impact of X-ray-producing BHs in low-mass galaxies on the epoch of reionization (e.g., Volonteri \& Gnedin 2009; Mirabel et al. 2011).

We thank the anonymous referee for helpful comments and suggestions. Support for A.E.R. was provided by NASA through Hubble Fellowship grant HST-HF2-51347.001-A awarded by the Space Telescope Science Institute, which is operated by the Association of Universities for Research in Astronomy, Inc., for NASA, under contract NAS 5-26555. A.E.R., J.E.G., and E.G. thank the Kavli Institute for Theoretical Physics for hosting the 2013 workshop "A Universe of Black Holes," during which this project began. This research was supported in part by the National Science Foundation under Grant No. NSF PHY11-25915. This research has made use of data obtained from the Chandra Data Archive and the Chandra Source Catalog, and software provided by the Chandra X-Ray Center (CXC) in the application packages CIAO, ChIPS, and Sherpa. This publication makes use of data products from the Wide-field Infrared Survey Explorer, which is a joint project of the University of California, Los Angeles, and the Jet Propulsion Laboratory/California Institute of Technology, funded by NASA. This work has also used observations made with the NASA Galaxy Evolution Explorer. GALEX is operated for NASA by the California Institute of Technology under NASA contract NAS5-98034. This study has made use of the NASA/IPAC Extragalactic Database (NED), which is operated by the Jet Propulsion Laboratory, California Institute of Technology, under contract with NASA. We are grateful to Michael Blanton and all who helped create 
the NASA-Sloan Atlas. Funding for the NASA-Sloan Atlas has been provided by the NASA Astrophysics Data Analysis Program (08-ADP08-0072) and the NSF (AST-1211644).

\section{APPENDIX \\ NOTES ON INDIVIDUAL SOURCES}

1. Mrk 361 (ObsID 6855; PI Komossa). Belongs to the field of view of the ultraluminous infrared galaxy III $\mathrm{ZW} 035$. To the best of our knowledge, this is the first paper reporting on the discovery of an X-ray source in the target dwarf galaxy.

2. NGC 855 (ObsID 7095; PI Swartz). Detection of a ULX in this dwarf galaxy was first reported by Swartz et al. (2008).

3. IC 399 (ObsID 9405; PI Gallagher). X-ray sources in this group dwarf galaxy were first reported by Desjardins et al. (2013). See also Tzanavaris et al. (2014).

4. UGC 4904 (ObsID 6729; PI Kulkarni). ToO observation to follow up on a core-collapse supernova in the target galaxy (Ofek et al. 2013). Analysis of the point-like X-ray population was first presented by Desjardins et al. (2014).

5. I Zw 18, Mrk 116 (ObsID 805; PI Bomans). A nuclear $\mathrm{X}$-ray source in this metal-poor dwarf galaxy was first reported by Ott et al. (2005). See also Kaaret et al. (2011), Kaaret \& Feng (2013), Prestwich et al. (2013), and Brorby et al. (2014).

6. UGC 5720 (ObsID 9519; PI Mas-Hesse). X-ray sources associated with this Ly $\alpha$-emitting galaxy were first reported by Grier et al. (2011). See also Mineo et al. (2012) and OtíFloranes et al. (2012).

7. Mrk 1434 (Obsid 3347; PI Barger). Belongs to the field of view of the Lockman Hole-Northwest (Yang et al. 2004). To the best of our knowledge, this is the first paper reporting on $\mathrm{X}$-ray sources associated with the target dwarf galaxy.

8. NGC 3413 (ObsID 7102; PI Swartz). The X-ray source in this galaxy is part of the Liu (2011) catalog (it is not, however, included in Swartz et al. 2008 or Swartz et al. 2009, as both studies focus on ULXs and thus discard nuclear X-ray sources).

9. UGC 06850 (ObsID 7135; PI Swartz). A bright X-ray source in this group dwarf galaxy was first reported by Swartz et al. (2009). See also Swartz et al. (2011).

10. SDSS J121326.02+543631.8 (ObsID 7071; PI Kaaret). Belongs to the field of view of NGC 4194 (Kaaret \& AlonsoHerrero 2008). To the best of our knowledge, this is the first paper reporting on an X-ray source associated with the target dwarf galaxy.

11. NGC 4214 (ObsID 5197; PI Zezas). X-ray sources in this nearby dwarf galaxy were first reported by Ghosh et al. (2006) and Leonidaki et al. (2010). See also Liu (2011) and Mineo et al. (2012).

12. VCC 344 (ObsID 9569; PI Zesaz). Belongs to the field of view of the nearby galaxy NGC 4261 (Worrall et al. 2010; see also Gültekin et al. 2009). To the best of our knowledge, this is the first paper reporting on X-ray sources in the target dwarf galaxy.

13. SDSS J122111.29+173819.1 (ObsID 8085; PI Treu). Belongs to the field of view of VCC 437 (Gallo et al. 2010). To the best of our knowledge, this is the first paper reporting on $\mathrm{X}$-ray sources in the target dwarf galaxy.

14. SDSS J122219.44+300344.1 (ObsID 7853; PI Mathur). Belongs to the field of view of NGC 4308 (Miller et al. 2012). To the best of our knowledge, this is the first paper reporting on an X-ray source in the target dwarf galaxy.

15. NGC 4395 (ObsID 5301; PI Moran). This nearby dwarf spiral galaxy harbors the least luminous Seyfert 1 nucleus known. O'Neill et al. (2006) first reported on the X-ray population resolved with Chandra.

16. SDSS J124517.25+270732.1 (Obsid 13928; PI Reines).

A. E. Reines et al., in preparation

17. NGC 4701 (ObsID 7148; PI Soria). Desroches \& Ho (2009) first reported on a nuclear X-ray source in this nearby late-type dwarf. Here we report on an additional off-nuclear source. See also Liu (2011).

18. NGC 4713 (ObsID 4019; PI Satyapal). Dudik et al. (2005) first reported on the lack of a nuclear X-ray source in this dwarf. Here we report on off-nuclear sources. See also Liu (2011).

19. NGC 5143 (ObbsID 4055; PI Sambruna). Belongs to the field of view of the FRI $1322+36$. To the best of our knowledge, this is the first paper reporting on X-ray sources in the target dwarf galaxy.

\section{REFERENCES}

Assef, R. J., Stern, D., Kochanek, C. S., et al. 2013, ApJ, 772, 26 Barth, A. J., Greene, J. E., \& Ho, L. C. 2008, AJ, 136, 1179

Becker, R. H., White, R. L., \& Helfand, D. J. 1995, ApJ, 450, 559 Blanton, M. R., \& Roweis, S. 2007, AJ, 133, 734

Brorby, M., Kaaret, P., \& Prestwich, A. 2014, MNRAS, 441, 2346

Condon, J. J., Cotton, W. D., Greisen, E. W., et al. 1998, AJ, 115, 1693

Conroy, C., Gunn, J. E., \& White, M. 2009, ApJ, 699, 486

Desjardins, T. D., Gallagher, S. C., Hornschemeier, A. E., et al. 2014, ApJ, 790, 132

Desjardins, T. D., Gallagher, S. C., Tzanavaris, P., et al. 2013, ApJ, 763, 121

Desroches, L.-B., \& Ho, L. C. 2009, ApJ, 690, 267

Dickey, J. M., \& Lockman, F. J. 1990, ARA\&A, 28, 215

Dudik, R. P., Satyapal, S., Gliozzi, M., \& Sambruna, R. M. 2005, ApJ, 620,113

Evans, I. N., Primini, F. A., Glotfelty, K. J., et al. 2010, ApJS, 189, 37

Fabbiano, G. 2006, ARA\&A, 44, 323

Feng, H., \& Soria, R. 2011, NewAR, 55, 166

Filippenko, A. V., \& Sargent, W. L. W. 1989, ApJL, 342, L11

Fruscione, A., McDowell, J. C., Allen, G. E., et al. 2006, Proc. SPIE, 6270, $62701 \mathrm{~V}$

Gallo, E., Treu, T., Jacob, J., et al. 2008, ApJ, 680, 154

Gallo, E., Treu, T., Marshall, P. J., et al. 2010, ApJ, 714, 25

Gehrels, N. 1986, ApJ, 303, 336

Ghosh, H., Mathur, S., Fiore, F., \& Ferrarese, L. 2008, ApJ, 687, 216

Ghosh, K. K., Rappaport, S., Tennant, A. F., et al. 2006, ApJ, 650, 872

Gilfanov, M. 2004, MNRAS, 349, 146

Gilfanov, M., Grimm, H.-J., \& Sunyaev, R. 2004, MNRAS, 347, L57

Greene, J. E. 2012, NatCo, 3, 1304

Greene, J. E., \& Ho, L. C. 2004, ApJ, 610, 722

Greene, J. E., \& Ho, L. C. 2007, ApJ, 670, 92

Grier, C. J., Mathur, S., Ghosh, H., \& Ferrarese, L. 2011, ApJ, 731, 60

Grimm, H.-J., Gilfanov, M., \& Sunyaev, R. 2002, A\&A, 391, 923

Grimm, H.-J., Gilfanov, M., \& Sunyaev, R. 2003, MNRAS, 339, 793

Gültekin, K., Cackett, E. M., Miller, J. M., et al. 2009, ApJ, 706, 404

Hao, C.-N., Kennicutt, R. C., Johnson, B. D., et al. 2011, ApJ, 741, 124

Ho, L. C. 2009, ApJ, 699, 626

Hong, J., van den Berg, M., Schlegel, E. M., et al. 2005, ApJ, 635, 907

Humphrey, P. J., \& Buote, D. A. 2008, ApJ, 689, 983

Izotov, Y. I., Guseva, N. G., Fricke, K. J., \& Henkel, C. 2014, A\&A, 561, AA33

Jarrett, T. H., Cohen, M., Masci, F., et al. 2011, ApJ, 735, 112

Jarrett, T. H., Masci, F., Tsai, C. W., et al. 2013, AJ, 145, 6

Kaaret, P., \& Alonso-Herrero, A. 2008, ApJ, 682, 1020

Kaaret, P., \& Feng, H. 2013, ApJ, 770, 20

Kaaret, P., Schmitt, J., \& Gorski, M. 2011, ApJ, 741, 10

Kennicutt, R. C., \& Evans, N. J. 2012, ARA\&A, 50, 531

Kormendy, J., \& Ho, L. C. 2013, ARA\&A, 51, 511

Kraft, R. P., Burrows, D. N., \& Nousek, J. A. 1991, ApJ, 374, 344

Lehmer, B. D., Alexander, D. M., Bauer, F. E., et al. 2010, ApJ, 724, 559

Lehmer, B. D., Berkeley, M., Zezas, A., et al. 2014, ApJ, 789, 52

Leonidaki, I., Zezas, A., \& Boumis, P. 2010, ApJ, 725, 842

Liu, J. 2011, ApJS, 192, 10 
McClintock, J. E., \& Remillard, R. A. 2006, in Compact Stellar X-ray Sources, ed. W. Lewin \& M. van der Klis (Cambridge: Cambridge Univ. Press), 157 Merloni, A., Heinz, S., \& di Matteo, T. 2003, MNRAS, 345, 1057

Miller, B., Gallo, E., Treu, T., \& Woo, J.-H. 2012, ApJ, 747, 57

Mineo, S., Gilfanov, M., \& Sunyaev, R. 2012, MNRAS, 419, 2095

Mirabel, I. F., Dijkstra, M., Laurent, P., Loeb, A., \& Pritchard, J. R. 2011, A\&A, 528, AA149

Moretti, A., Campana, S., Lazzati, D., \& Tagliaferri, G. 2003, ApJ, 588,696

Natarajan, P. 2014, GReGr, 46, 1702

Ofek, E. O., Fox, D., Cenko, S. B., et al. 2013, ApJ, 763, 42

O’Neill, P. M., Kaspi, S., Laor, A., et al. 2006, ApJ, 645, 160

Otí-Floranes, H., Mas-Hesse, J. M., Jiménez-Bailón, E., et al. 2012, A\&A, 546, A65

Ott, J., Walter, F., \& Brinks, E. 2005, MNRAS, 358, 1423

Pellegrini, S. 2010, ApJ, 717, 640

Plotkin, R. M., Gallo, E., Miller, B. P., et al. 2014, ApJ, 780, 6

Prestwich, A. H., Tsantaki, M., Zezas, A., et al. 2013, ApJ, 769, 92

Reines, A. E., \& Deller, A. T. 2012, ApJL, 750, L24

Reines, A. E., Greene, J. E., \& Geha, M. 2013, ApJ, 775, 116

Reines, A. E., Plotkin, R. M., Russell, T. D., et al. 2014, ApJL, 787, L30
Reines, A. E., Sivakoff, G. R., Johnson, K. E., \& Brogan, C. L. 2011, Natur, 470, 66

Schramm, M., Silverman, J. D., Greene, J. E., et al. 2013, ApJ, 773, 150

Soria, R., Fabbiano, G., Graham, A. W., et al. 2006, ApJ, 640, 126

Stern, D., Assef, R. J., Benford, D. J., et al. 2012, ApJ, 753, 30

Swartz, D. A., Soria, R., \& Tennant, A. F. 2008, ApJ, 684, 282

Swartz, D. A., Soria, R., Tennant, A. F., \& Yukita, M. 2011, ApJ, 741, 49

Swartz, D. A., Tennant, A. F., \& Soria, R. 2009, ApJ, 703, 159

Tzanavaris, P., Gallagher, S. C., Hornschemeier, A. E., et al. 2014, ApJS, 212,9

Volonteri, M. 2010, A\&ARv, 18, 279

Volonteri, M., \& Gnedin, N. Y. 2009, ApJ, 703, 2113

Worrall, D. M., Birkinshaw, M., O’Sullivan, E., et al. 2010, MNRAS, 408, 701

Wright, E. L., Eisenhardt, P. R. M., Mainzer, A. K., et al. 2010, AJ, 140, 1868

Xia, X. Y., Xue, S. J., Mao, S., Boller, T., Deng, Z. G., \& Wu, H. 2002, ApJ, 564, 196

Yang, Y., Mushotzky, R. F., Steffen, A. T., Barger, A. J., \& Cowie, L. L. 2004, AJ, 128, 1501

Zhang, W. M., Soria, R., Zhang, S. N., Swartz, D. A., \& Liu, J. F. 2009, ApJ, 699,281

Zhao, P., Grindlay, J. E., Hong, J. S., et al. 2005, ApJS, 161, 429 